\title{
Abordagem metodológica baseada em imagens do SAR-R99B para identificar prováveis pistas de pouso não-homologadas na Amazônia.
}

\author{
Sergio Alexandre Saloio ALVES ${ }^{1}$, Luis Ricardo ASSANTE², Edson Eyji SANO33, Paulo Roberto MENESES ${ }^{4}$ \\ RESUMO \\ Este estudo apresenta uma abordagem metodológica baseada em imagens de radar e nos critérios de tonalidade, tamanho \\ e forma geométrica para identificar prováveis pistas de pouso não-homologadas na Amazônia. Os seguintes procedimentos \\ foram conduzidos: georreferenciamento da imagem do sensor SAR-R99B do município paraense de Itaituba, adquirida na \\ banda L, polarização HH e resolução espacial de três metros; subtração do ruído speckle com filtro mediana; classificação com \\ a técnica não-supervisionada ISODATA; vetorização da classe indicativa dos alvos de interesse; e cálculo e seleção automática \\ dos alvos de interesse por critérios de índice de circularidade e de tortuosidade. Foram identificados dez alvos, dos quais dois \\ foram considerados como prováveis pistas de pouso pelos referidos índices.
}

PALAVRAS-CHAVE: Radar, sensoriamento remoto, índice de circularidade, índice de tortuosidade.

\section{Methodological approach based on SAR-R99B radar images to identify potential unofficial airplane strips in Amazonia}

\section{ABSTRACT}

This study presents a methodological approach based on radar images and on gray level, size and geometric pattern to identify potential unofficial airplane strips in Amazonia. The following procedures were conducted: georeferencing of a L-band, $\mathrm{HH}$ polarization, 3-meter spatial resolution image obtained by the SAR-R99B system over the Itaituba municipality, State of Para; subtraction of speckle noise with a median filter; image classification with the ISODATA unsupervised technique; conversion of selected targets of interest into vector format; and calculation and automatic selection of targets of interest by the circularity and tortuosity indices. Among the 10 previously identified targets, two were considered as potential unofficial airplane strips by the above mentioned indices.

KEY WORDS: Radar, remote sensing, circularity index, tortuosity index.

\footnotetext{
${ }^{1}$ Comando da Aeronáutica. E-mail: ssaloio@ig.com.br

${ }^{2}$ Ministério da Defesa. E-mail: luis.assante@defesa.gov.br

${ }^{3}$ Embrapa Cerrados. E-mail: sano@cpac.embrapa.br

${ }^{4}$ Universidade de Brasília E-mail: pmeneses@unb.br
} 
O crime organizado tem construído e utilizado pistas de pouso não-homologadas na Amazônia para burlar a fiscalização e o controle governamental do comércio ilegal de drogas, armas e biodiversidade amazônica. As pistas de pouso não-homologadas na Amazônia são preparadas para permitir a corrida de aeronaves de pequeno porte, isto é, monomotores com comprimento em torno de $7 \mathrm{~m}$, envergadura de $11 \mathrm{~m}$ e peso médio de $500 \mathrm{~kg}$. O comprimento mínimo da pista necessário para o pouso desse tipo de aeronave é de $300 \mathrm{~m}$, com largura média de $20 \mathrm{~m}$.

Imagens de sensoriamento remoto com alta resolução espacial são as mais indicadas para a identificação de tais alvos. Para a Amazônia, os sensores de radar são mais promissores do que os sensores ópticos devido à persistente cobertura de nuvens durante a maior parte do ano. No entanto, cenas de radar com resoluções espaciais da ordem de três metros estão disponíveis somente a nível de aeronave. No Brasil, desde 2005, os sensores de radar de abertura sintética (SAR), embarcados nas aeronaves R99B da Força Aérea Brasileira, vêm adquirindo dados em diferentes regiôes do País e da América do Sul. Esse sensor, denominado de SAR-R99B, opera na banda L (comprimento de onda de $23 \mathrm{~cm}$ ), com múltipla polarização (HH, VV, HV, VH) e na banda X (comprimento de onda de $3 \mathrm{~cm}$ ), com polarização $\mathrm{HH}$. As imagens podem ser obtidas com resoluçôes espaciais de $3 \mathrm{~m}, 6 \mathrm{~m}$ ou $18 \mathrm{~m}$, com uma respectiva faixa de recobrimento no terreno de $10 \mathrm{~km}, 20 \mathrm{~km}$ e $60 \mathrm{~km}$. Devido ao curto período de funcionamento, poucos trabalhos de avaliação desses dados de radar estão disponíveis na literatura. Os primeiros resultados estão sendo divulgados em anais de simpósios e congressos em diferentes áreas de aplicação, tais como em monitoramento de desflorestamento (Zaloti Jr. Et al., 2006) e em geologia (Almeida et al., 2007). O objetivo deste estudo foi analisar o potencial das imagens do sensor SAR-R99B para a detecção de prováveis pistas de pouso não-homologadas na Amazônia.

A área de estudo, com uma dimensão em torno de $36 \mathrm{~km}^{2}$, situou-se no município de Itaituba, Pará, entre as latitudes $07^{\circ} 03^{\prime} 35^{\prime \prime}$ e $07^{\circ} 06^{\prime} 52^{\prime \prime}$ e entre as longitudes 56 $53^{\prime} 41^{\prime \prime}$ e $56^{\circ} 50^{\prime} 24^{\prime \prime}$. Nessa área, caracterizada pela presença de uma alta concentração pistas de pouso não-homologadas, foi obtida, em outubro de 2005, uma imagem SAR-R99B na banda $\mathrm{L}$, polarização $\mathrm{HH}$, resolução espacial de $3 \mathrm{~m}$ e ângulo de incidência variando de $51^{\circ}$ a $64^{\circ}$.

A imagem foi georreferenciada para o sistema de projeção UTM, datum SAD69 e processada com filtro passa-baixa do tipo mediana e tamanho de $3 \times 3$ pixels (Figura 1a). Em seguida, a imagem foi classificada pelo método nãosupervisionado ISODATA, um classificador que agrupa pixels com base no centro de classes medidas por uma distância estatística. Foi definido um número mínimo de quatro classes, número máximo de sete classes, cinco iterações e um mínimo de 100 pixels por classe. A classe com tons de cinza mais escuros foi considerada como indicadora de pistas de pouso, pois, em uma imagem de radar, os solos expostos secos e com rugosidade lisa ou cobertos com vegetação herbácea, que são os materiais tipicamente encontrados numa pista de pouso não-homologada, apresentam, em sua maioria, valores baixos de retroespalhamento. Um conjunto formado por 3.085 polígonos, com áreas e formas variadas, foi classificado como pertencentes a essa classe. Esses polígonos foram então vetorizados e convertidos para o formato shape. Para identificar os polígonos que se aproximavam das dimensões e formas de uma pista de pouso, foi desenvolvido um algoritmo em linguagem de programação IDL para calcular a área e o perímetro correspondentes a todos os polígonos convertidos em formato shape (Souza, 2006). Com base nas dimensōes mínimas e máximas que uma pista de pouso não-homologada na região amazônica deve apresentar, o algoritmo acessa a tabela de vetores do shapefile e seleciona apenas os que satisfazem às medições desejadas. Dez polígonos com os parâmetros estabelecidos foram identificados, os quais foram tidos como os mais prováveis de serem pistas de pouso (Figura 1b).

$\mathrm{O}$ algoritmo calcula ainda um índice de circularidade (CR - circularity ratio, Selkirk, 1982) para os polígonos selecionados. Índice de circularidade é definido como $\mathrm{CR}=$ $4 \pi \mathrm{A} / \mathrm{p}^{2}$, em que $\mathrm{A}$ e $\mathrm{p}$ correspondem, respectivamente, à área e ao perímetro de um polígono. Os valores de CR variam no intervalo de 0 a 1 . Valores próximos de 0 indicam que o polígono possui forma mais estreita e alongada, ou seja, próximo de uma pista de pouso, enquanto valores próximos de 1 indicam que o polígono possui a forma de um círculo. Das dez áreas selecionadas anteriormente, os polígonos 2, 7 , 8 e 9 apresentaram os valores de CR mais baixos $(\mathrm{CR}<0,11$; Tabela 1).

Dependendo da combinação entre valores de área e de perímetro, um polígono com potencial baixo de ser uma pista de pouso pode apresentar valor de CR relativamente baixo. Este é o caso, por exemplo, do polígono 9, que pode ser visualmente descartado por causa da sua acentuada irregularidade geométrica, mas que possui CR relativamente baixo. Neste sentido, foi incluído um outro índice, o de tortuosidade, definido por $\mathrm{T}=2 \mathrm{~d} / \mathrm{p}$, em que $\mathrm{d}$ corresponde à distância longitudinal média do polígono. Quanto maior $\mathrm{o}$ valor de $\mathrm{T}$ de um polígono, maior a chance de corresponder a uma pista de pouso. Com este critério, os polígonos 2 e 8 , com valores de $\mathrm{T}$ de 0,792 e 0,942 (Tabela 1 ), respectivamente, foram identificados como prováveis pistas de pouso, os demais representando áreas de sombreamento ou espelho de água. Esses dois alvos não estavam catalogados no ROTAER, manual que possui as informaçóes aeronáuticas do Brasil.

Os resultados deste trabalho comprovam que as imagens de radar de alta resolução da FAB constituem-se numa boa opção 
(A)

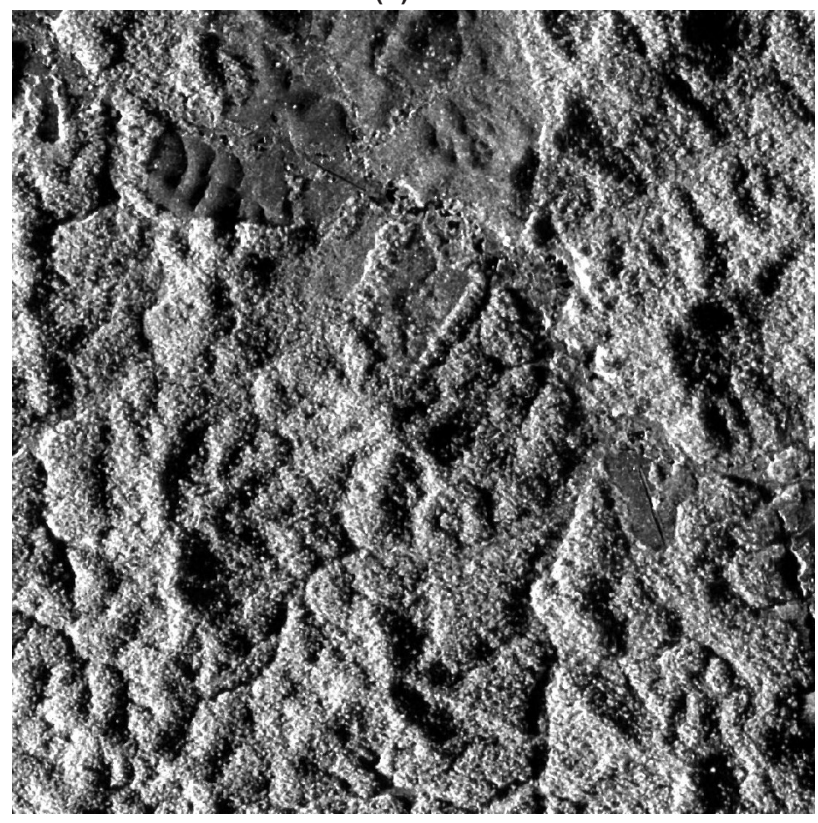

(B)

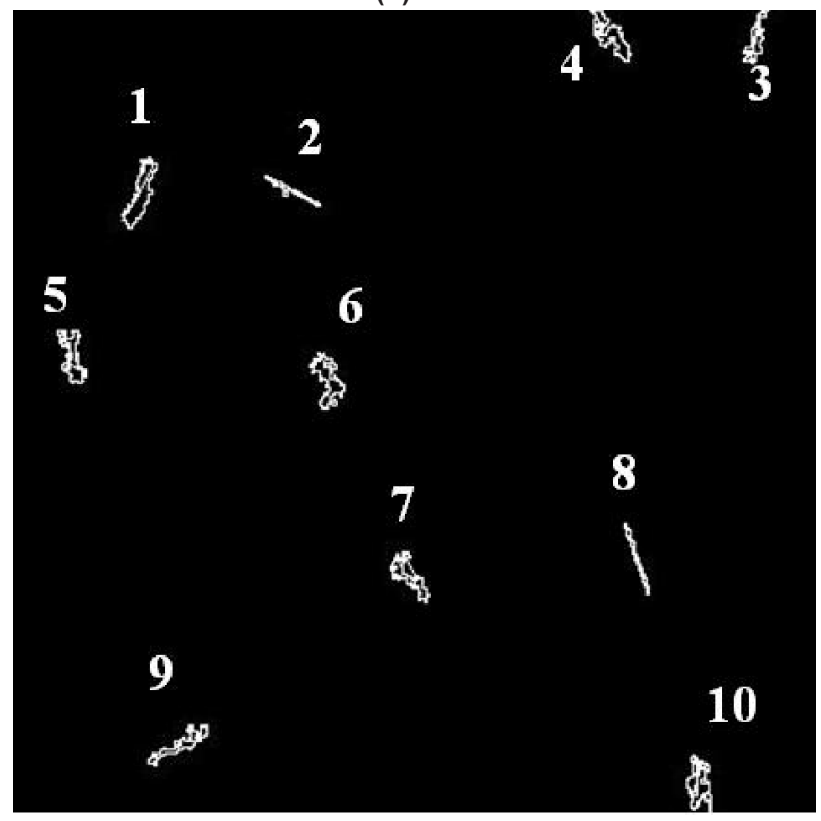

Figura 1 - (a) Imagem de radar da área de estudo, após processamento com filtro mediana; (b) Identificação, por critérios de dimensões típicas, de dez possíveis pistas de pouso não-homologadas. Os alvos 2 e 8 foram selecionados como prováveis pistas de pouso pelos critérios de índices de circularidade e de tortuosidade.
Tabela 1 - Área, perímetro, índice de circularidade (CR) e índice de tortuosidade $(\mathrm{T})$ correspondentes aos dez polígonos selecionados na área de estudo como prováveis pistas de pouso.

\begin{tabular}{lcccc}
\hline Alvo & Área $\left(\mathrm{m}^{2}\right)$ & Perímetro $(\mathrm{m})$ & $\mathrm{CR}$ & $\mathrm{T}$ \\
\hline 1 & 41605 & 1607 & 0,202 & 0,630 \\
2 & 8920 & 1114 & 0,090 & 0,792 \\
3 & 20325 & 1498 & 0,114 & 0,535 \\
4 & 34740 & 1657 & 0,159 & 0,543 \\
5 & 30989 & 1497 & 0,174 & 0,531 \\
6 & 33558 & 1877 & 0,120 & 0,394 \\
7 & 22648 & 1637 & 0,106 & 0,465 \\
8 & 9930 & 1089 & 0,105 & 0,942 \\
9 & 22043 & 1643 & 0,103 & 0,593 \\
10 & 33135 & 1713 & 0,142 & 0,474 \\
\hline
\end{tabular}

para a identificação de prováveis pistas de pouso clandestinas na região amazônica. $\mathrm{O}$ método proposto consiste na utilização de uma técnica de processamento digital bastante conhecida pela comunidade científica e por usuários de sensoriamento remoto e de algoritmos de formulaçôes matemáticas simples e que podem ser aplicados de forma automática, num tempo muito rápido.

\section{BIBLIOGRAFIA CITADA}

Almeida, T.; Meneses, P.R.; Araújo Filho, J.O. 2007. As imagens do SAR multipolarimétrico R99B-SIPAM na caracterização de fácies e estruturas da suíte Pedra Branca (maciço de Mocambo e Pedra Branca, Nova Roma-GO) In: Simpósio Brasileiro de Sensoriamento Remoto, 13, Florianópolis, SC. Anais... São José dos Campos, INPE, p.1443-1450.

Selkirk, K.E. 1982. Pattern and place. An introduction to the mathematics of geography. Cambridge University Press, Cambridge, UK, 55 pp.

Souza, P.E.U. 2006. Detecção de polígonos com geometria similar a aeródromos. Brasília, comunicação pessoal.

Zaloti Jr., O.D.; Gonçalves, F.G.; Freitas, C.C.; Santanna, S.J.S.; Santos, J.R. 2006. Evaluating the potential of SAR-R99B L- and $\mathrm{X}$-bands data for Amazon deforestation increment mapping. In: International Geoscience and Remote Sensing Symposium (IGARSS06), Denver, CO, USA. Proceedings... São José dos Campos, IEEE, p. 2662-2665.

Recebido em 29/11/2009

Aceito em 04/06/2009 
